\title{
Formation and Evolution of the Quark-Gluon Plasma
}

\author{
Jean Letessier ${ }^{1}$, Johann Rafelski ${ }^{1,2}$ and Ahmed Tounsi ${ }^{1}$ \\ ${ }^{1}$ Laboratoire de Physique Théorique et Hautes Energies* \\ Université Paris 7, 2 place Jussieu, F-75251 Cedex 05. \\ 2 Department of Physics, University of Arizona, Tucson, AZ 85721 \\ and Theory Division, CERN, 1211 Geneva 23, Switzerland
}

\begin{abstract}
Imposing an equilibrium between the thermal pressure of deconfined quarks and gluons and the dynamical compression pressure exercised by in-flowing nuclear matter, we study the initial thermal conditions reached in a quark-gluon plasma fireball formed in a relativistic heavy ion collision. We show that entropy is produced primarily in the pre-equilibrium stage of the reaction. We test our approach, comparing our results with the $\mathrm{S} \rightarrow \mathrm{W} / \mathrm{Pb}$ collision results at $200 \mathrm{GeV}$ A and find a surprising degree of agreement assuming about 50\% stopping. We apply our method to a determination of the conditions in collisions of $\mathrm{Au} \rightarrow \mathrm{Au}$ at $11 \mathrm{GeV} \mathrm{A}$ and $\mathrm{Pb} \rightarrow \mathrm{Pb}$ at $157 \mathrm{GeV} \mathrm{A}$, assuming full stopping of momentum, energy and baryon number. Our detailed results directly determine the spectral shape and abundance of (strange) hadrons and electromagnetic probes (photons, dileptons) produced in the collision, and we explore specific experimental consequences.
\end{abstract}

Published in Phys. Lett. B333 (1994) 484

CERN-TH.7304/94

PAR/LPTHE/94-23

May 1994

${ }^{*}$ Unité associée au CNRS UA 280. 
J. Letessier, J. Rafelski and A. Tounsi

\section{Introduction}

Our objective is to obtain a simple and qualitative understanding of the initial conditions reachable in high-energy collisions of nuclei. Although the information about the high density state that we observe using hadronic probes is originating in the late stages of the evolution of the high-density matter formed in relativistic nuclear collisions, some basic physical principles will allow us to go back in time in order to obtain a rather precise picture of the initial state. These considerations allow us to determine the expected formation rates of electromagnetic probes of the dense state (dileptons, photons), which provide an important verification of our understanding of the structure of high-density matter. In our present investigation, which is based on the hypothesis that the quark-gluon deconfined phase has been formed in the interaction, we shall use a slight generalization to chemical (particle abundance) nonequilibrium of the quark-gluon plasma (QGP) equations of state described by us previously. These incorporate perturbative QCD interaction effects and thermal particle masses [1]. We have already shown [2, 3, 4] that the formation of the QGP phase is indeed, at the highest now available energy of $200 \mathrm{GeV}$ A, the simplest hypothesis capable to consistently account for all experimental data. At the lower end (10-15 GeV A) of the energy range considered here, this QGP hypothesis is not inevitable, given the present-day data sample [5]. The key step we make in the present work is to establish, based on very plausible and simple dynamical conditions, the early values of temperature and chemical potentials, which in our previous work arose from the study of experimental results.

Implicit in the physical picture employed here (seeRefs. [3, 4] for more details), is that a space-time region of hot, dense hadronic matter with nearly thermal properties is formed in the centre of momentum (CM) frame ('central region'), which can be characterized by the statistical parameters, the temperature $T$ and the baryo-chemical potential $\mu_{\mathrm{B}}=3 \mu_{\mathrm{q}}$ or equivalently the quark fugacity $\lambda_{\mathrm{q}}=\exp \left(\mu_{\mathrm{q}} / T\right)$, and similarly for strange quarks $\lambda_{\mathrm{s}}=\exp \left(\mu_{\mathrm{s}} / T\right)$. In addition, we will study the approach to chemical equilibrium (particle-abundance equilibrium) by all different components. We thus introduce the chemical occupancy factors of the different particles: gluons $\gamma_{\mathrm{G}}$, light quarks and antiquarks $\gamma_{\mathrm{q}}$, and strange quarks $\gamma_{\mathrm{s}}$. When the accessible phase space is saturated we have $\gamma_{i}=1$ and the chemical equilibrium is established. Note that the $q, \bar{q}$ abundance is controlled by the two parameters $\lambda_{\mathrm{q}}$ and $\gamma_{\mathrm{q}}$ and thus the number of both can be established independently: the fugacity factor $\lambda_{\mathrm{Q}}=\gamma_{\mathrm{q}} \lambda_{\mathrm{q}}$ determines the quark abundance, and the factor $\lambda_{\overline{\mathrm{Q}}}=\gamma_{\mathrm{q}} \lambda_{\mathrm{q}}^{-1}$ determines the antiquark abundance. We assume that thermalization is faster than the chemical equilibration - but also note that the mechanism causing the rapid formation of a thermal particle distribution remains little understood. Studying final particle spectra it can be convincingly argued in favour of thermalization. An extensive analysis [6] has been carried out accounting for diverse distortions of the spectra caused by the disintegration of unstable hadrons and longitudinal flow. These effects in the transverse mass $m_{\perp}$ particle spectra are small at high $m_{\perp}$; in the domain $m_{\perp}>1.5 \mathrm{GeV}$ one can directly infer for $\mathrm{S}-\mathrm{W} / \mathrm{Pb}$ collisions at $200 \mathrm{GeV}$ A a source temperature of about $232 \pm 5 \mathrm{MeV}$ in the spectra of several strange-particles [7] as well as in the spectra of $\pi^{0}$ and $\eta$ [8].

Let us briefly summarize the four arguments pointing to the QGP nature of the dense fireball formed in collisions at $200 \mathrm{GeV} \mathrm{A}$ : 
i) Strange-quark fugacity: $\lambda_{\mathrm{S}} \sim 1$ is natural for a directly disintegrating QGP phase where the symmetry between the $s$ and $\bar{s}$ quarks is reflected naturally in the value of $\lambda_{\mathrm{s}}=1$. In the confined phase, whatever the equation of state, $\lambda_{\mathrm{s}}=1$ is an exceptional condition at finite baryon density, since strange baryons (carriers of $s$-quarks) and mesons (carriers of $\bar{s}$-quarks) have different masses. Studying the relative abundance of strange-particles it is possible to precisely determine the final-state value of $\lambda_{\mathrm{s}}$. The analysis of the $\mathrm{S} \rightarrow \mathrm{W}$ [3, 9] and $\mathrm{S} \rightarrow \mathrm{S}$ [10] data has shown that the strange-quark fugacity $\lambda_{\mathrm{s}} \simeq 1$. The recently reported $\bar{\Omega} / \Omega$ result [11] has provided further independent evidence [3] that $\lambda_{\mathrm{s}} \simeq 1$. Note that one finds, for the lower energy AGS results obtained with 14.6 GeV A projectiles [5, 12, a value $\lambda_{\mathrm{s}}=1.72 \pm 0.19$, distinctly different from unity.

ii) Strange-quark phase-space occupancy: Comparing the abundance of particles with unequal numbers of strange-quarks, one can obtain a measure of the strange-quark phasespace occupancy, and at $200 \mathrm{GeV} \mathrm{A}$ one finds $\gamma_{\mathrm{s}} \rightarrow 1$, suggesting that rather effective processes (presumably gluon-based [13]) were available to produce strangeness [3, 9, 10].

iii) Excess particle multiplicity/entropy: The flow of charged hadrons can be used [2] to establish the hadronic multiplicity produced per participating baryon, and this quantity can be related to the entropy produced in the interaction. This entropy content is easier to bring into consistency with the QGP picture than with a more conservative reaction picture in which colour is frozen, thus, when other conditions are equal, less entropy is produced.

iv) Consistency with QGP equations of state: Using the QGP equations of state one finds [价 that the temperature in $m_{\perp}$ spectra and $\lambda_{\mathrm{q}}$, as determined using strange-particle multiplicity, are consistent with the energy content available in the collision.

In this work we will be able to go beyond this last observation and to determine the observable values of $T, \lambda_{\mathrm{q}}$ alone on the basis of the CM collision energy and its stopping. We will thus determine:

- the primordial temperature for the $\mathrm{S} \rightarrow \mathrm{W} / \mathrm{Pb}$ collisions at $200 \mathrm{GeV} \mathrm{A}\left(E_{\mathrm{CM}} \simeq 8.8 \mathrm{GeV}\right)$, and the conditions:

- prevailing in collisions $\mathrm{Si} \rightarrow \mathrm{Au}$ at $14.6 \mathrm{GeV} \mathrm{A}, \mathrm{Au} \rightarrow \mathrm{Au}$ at $11 \mathrm{GeV} \mathrm{A}\left(E_{\mathrm{CM}} \simeq 2.6 \mathrm{GeV}\right)$,

- expected for the forthcoming $\mathrm{Pb} \rightarrow \mathrm{Pb}$ collisions at $157 \mathrm{GeV} \mathrm{A}\left(E_{\mathrm{CM}} \simeq 8.6 \mathrm{GeV}\right)$.

In principle all our results are now fully ab initio and do not contain any parameters, apart from the assumtion that, for $\mathrm{S} \rightarrow \mathrm{W} / \mathrm{Pb}$, the stopping $\eta_{i}$ of energy, momentum and baryon number is about $50 \%$ [14, while we take $\eta_{i} \simeq 1$ in the other cases considered above.

From the relaxation-time constants applicable to the strange-quark production [13], which is, without doubt, slower than for gluons and light quarks, one can infer that the time $t=t_{\mathrm{s}}=3-5 \mathrm{fm} / \mathrm{c}$. At this time (nearly) complete chemical equilibrium is reached and the temperature has cooled to its observable value $\left.T\right|_{t_{\mathrm{s}}} \equiv T_{0}$. Here $t_{\mathrm{s}}$ is about a factor $6-10$ longer than what is found for the relaxation time of the chemical equilibration of light quarks and gluons [15, 16]. Correspondingly, we shall assume that in the epoch $\left(t=t_{\mathrm{ch}} \simeq 1 \mathrm{fm} / c\right)$ of the hot matter evolution, when the quark and gluon chemical equilibrium characterized by phase-space occupancy $\left.\left.\gamma_{\mathrm{q}}\right|_{t_{\mathrm{ch}}} \simeq \gamma_{\mathrm{G}}\right|_{t_{\mathrm{ch}}} \simeq 1$ is reached, the occupancy of the strange quark phase-space is about $\left.\gamma_{\mathrm{s}}\right|_{t_{\mathrm{ch}}}=0.15$. We also presume that the initial thermalization time $t=t_{\mathrm{th}}$, for quarks and gluons already made, is faster than the chemical equilibration. We will take the chemical occupancy factor $\left.\left.\gamma_{\mathrm{q}}\right|_{t_{\mathrm{th}}} \simeq \gamma_{\mathrm{G}}\right|_{t_{\mathrm{th}}} \simeq 0.2,\left.\gamma_{\mathrm{s}}\right|_{t_{\mathrm{th}}}=0.03$ when considering the very initial conditions of thecentral fireball. It is not possible to really start with all 
$\gamma_{i}=0$ since even in the pre-thermal phase a number of quark pairs and gluons will be produced. On the other hand, the exact values of $\gamma_{i}$ do not matter since conservation laws and other constraints almost fully determine the system. Thus any other 'reasonable' initial choice would in no way alter the results we present here.

The energy (and baryon number) required in the fireball is drawn from the fraction $\eta_{\mathrm{E}}$ of in-flowing energy and the fraction $\eta_{\mathrm{B}}$ of in-flowing baryon number, which is contributing to the thermal central fireball. These 'stopping parameters' characterize the phenomenon of compression and conversion of energy from relative motion into the thermal energy content of the central fireball, and ultimately into the final particle multiplicity. It is important to notice that the duration of the collision is considerable on the scale applied here. A large nucleus has a diameter of $\simeq 12 \mathrm{fm}$ and at the CM energy per nucleon available today, up to $9.6 \mathrm{GeV}$ A, the Lorenz contraction factor $\left(E_{\mathrm{cm}} / m \simeq 10\right)$ implies that the collision time $t_{\text {col }} \geq 1 \mathrm{fm} / c$. The parton cascades [15] (albeit for higher energies) and QCD gluon multiplication rates [16] place the time at which the chemical equilibrium of light quarks and gluons is reached at about $t_{\mathrm{ch}}=0.5-1 \mathrm{fm} / c$ in agreement with what one could infer from simple perturbative considerations of QCD cross sections. Thus for present-day heavy-ion collisions $\left(E_{\text {beam }} \leq 200 \mathrm{GeV} \mathrm{A}\right.$ ) the thermalization and chemical equilibrium of quarks and gluons is reached while the nuclear collision is taking place. During this collision period, the pressure exercised on the central fireball by the impacting matter is nearly constant. Therefore the degree of compression of the central fireball can be found by equating the dynamical pressure associated with the kinetic motion of the colliding nuclei, with the dominant resisting force, which is the internal thermal pressure of the fireball. Moreover, since the chemical equilibration of quarks and gluons occurs during the collision, it also occurs in condition of constant pressure.

Once the collision is terminated the central fireball approached the chemical equilibrium of light quarks and gluons at some constant pressure will commence to cool down by producing the strange-quark pairs and also by expanding in all directions. Let us first imagine that there is no strangeness production. Then the expansion of a nearly ideal quantum gas would occur without production of additional entropy, considering a hydrodynamic expansion of a locally equilibrated system. Consequently, the specific entropy per baryon $S / B$ will remain nearly constant, even in the presence of considerable particle evaporation: such emission processes are likely to reduce thebaryon and entropy content of the fireball at comparable rate. Since the specific entropy is dimensionless, it can only depend on dimensionless quantities, and for chemically equilibrated system with negligible strangeness fraction this can only be $\lambda_{\mathrm{q}}=\exp \left(\mu_{\mathrm{q}} / T\right)$. Consequently, this expansion of the fireball in the vacuum will occur at fixed $\lambda_{\mathrm{q}}$. This situation is in our opinion little changed if one considers the production of strange pairs; the entropy of the non-strange fraction will remain nearly constant for the reasons described, and thus again $\lambda_{\mathrm{q}}=$ const. The total entropy increases by the amount produced making strange-quarks, while the energy transfer to the strange-quark gas reduces the speed of the collective expansion [17].

We will next discuss the dynamical pressure in the collision, and review, in the following, in more quantitative manner the time scenario described here, including a discussion of the pertinent statistical fireball properties as well as the evolution of the entropy in the nuclear reaction. Some experimental consequences will also be studied. 


\section{Pressure balance}

We now consider quantitatively the balance between the dynamical compression exercised by the in-flowing matter and the resisting thermal pressure of the constituents of the central fireball. The pressure due to kinetic motion follows from well-established principles, and can be directly inferred, e.g. from Eq. (15) of Ref. [18] for the energy-momentum tensor:

$$
T^{i j}(x)=\int p^{i} u^{j} f(x, p) \mathrm{d}^{3} p, \quad i, j=1,2,3,
$$

where $u^{j}=p^{j} / E$. We take for the phase-space distribution of incident particles $f(x, p)=$ $\rho_{0}(x) \delta^{3}\left(\vec{p}-\vec{p}_{\mathrm{CM}}\right)$. To obtain the pressure exercised by the flow of matter, we consider the pressure component $T^{j j}$, with $j$ being the direction of $\vec{v}_{\mathrm{CM}}$. The result is:

$$
P_{\mathrm{dyn}}=\eta_{\mathrm{p}} \frac{p_{\mathrm{CM}}^{2}}{E_{\mathrm{CM}}} \rho_{0}
$$

Here it is understood that the energy $E_{\mathrm{CM}}$ and momentum $P_{\mathrm{CM}}$ is given in the CM frame, and per nucleon. We have introduced in Eq. (2) the momentum stopping fraction $\eta_{\mathrm{p}}$ - only this fraction $0 \leq \eta_{\mathrm{p}} \leq 1$ of the incident CM momentum can be used by a particle incident on the central fireball (the balance remains in longitudinal, unstopped motion) in order to exercise dynamical pressure. For a target transparent to the incoming flow, there would obviously be no pressure exercised. In principle, Eq. (2) defines the momentum stopping $\eta_{\mathrm{p}}$ and it is our hope and expectation that this $\eta_{\mathrm{p}} \simeq \eta_{\mathrm{E}}$, the latter being defined by studying the fraction of the original beam energy found in the transverse direction to the beam motion [14]. The magnitude of momentum (energy) stopping has been studied for different reactions. In the $\mathrm{S} \rightarrow \mathrm{W} / \mathrm{Pb}$ reactions at $200 \mathrm{GeV} \mathrm{A}$, the energy stopping is $\eta_{\mathrm{E}} \simeq 0.5$, and we therefore assume a similar value for our parameter $\eta_{\mathrm{p}}$. At lower energies we will move towards full stopping, that is $\eta_{i}=1$. We also anticipate that $\eta_{i}=1$ when we study collisions of largest nuclei such as $\mathrm{Pb} \rightarrow \mathrm{Pb}$.

Note that the magnitude of the dynamical pressure can be obtained qualitatively as follows: assume that only a fraction $0 \leq \eta_{\mathrm{p}} \leq 1$ of the incident CM momentum can be used by a particle incident on the central fireball in order to exercise pressure. We divide this change in momentum per increment in time and surface to obtain the pressure, converting the arriving particles into impact density and, taking also $d t=d z / v_{\mathrm{CM}}$, we find again Eq. (2). It is interesting to note that (with full stopping and using the normal nuclear density, $\rho_{0}=0.16 \mathrm{fm}^{-3}$ ) one finds the benchmark value $P_{\text {dyn }}=0.5 \mathrm{GeV} / \mathrm{fm}^{3}$ (respectively 1 $\mathrm{GeV} / \mathrm{fm}^{3}$ ) at $\gamma_{\mathrm{CM}}=3.4$ (respectively 6.4 ). In a symmetric collision this corresponds to an incident projectile at $23.6 \mathrm{GeV} / \mathrm{c}^{2} \mathrm{~A}$ (respectively $=86.3 \mathrm{GeV} / \mathrm{c}^{2} \mathrm{~A}$ ).

The idea of deconfinement and QGP is to some extent based on the simple picture of a confined hadron in which the internal Fermi pressure of constituents is balanced by the external vacuum pressure, which has the magnitude $\mathcal{B} \simeq 0.1 \mathrm{GeV} / \mathrm{fm}^{3}$. Applying the same idea to the determination of the initial conditions in the collision, we demand that the internal thermal (and Fermi) pressure $P_{\mathrm{th}}$ be balanced by the dynamical and vacuum pressure. We 
thus obtain the condition:

$$
P_{\mathrm{th}}(T, \ldots)=P_{\mathrm{dyn}}+P_{\mathrm{vac}}=\eta_{\mathrm{p}} \frac{E_{\mathrm{CM}}^{2}-m^{2}}{E_{\mathrm{CM}}} \rho_{0}+\mathcal{B} .
$$

The QGP equations of state will determine the left-hand side of Eq. (3). These equation follow from a slight generalization of our previous work [1], as we now allow for the absence of chemical equilibrium of gluons and light quarks. In short, we take ideal quantum gases and shall allow for the suppression of the occupancy of gluons $G$ and all quarks by the factors $\gamma_{i}, i=q, s, G$. We will allow for 'thermal' masses of all these particles with $m_{i}^{2}=m_{0}^{2}+(c T)^{2}$. In principle we have $c^{2} \propto \alpha_{\mathrm{s}}$, but given the current uncertainty regarding the value of the coefficient $c$ we shall simply explore its consequence in the domain $c \sim 2$, arising for $\alpha_{s} \sim 1$ in the standard formulas. We take $m_{\mathrm{q}}^{0}=5 \mathrm{MeV}, m_{\mathrm{s}}^{0}=160 \mathrm{MeV}$, and $m_{\mathrm{G}}^{0}=0$. We correct the quantum degeneracy factors using the perturbative thermal QCD corrections according to the prescription of Ref. [1]. For the QCD coupling, we take $\alpha_{s}=0.6$. Numerical integration of the Bose/Fermi distributions for quarks/gluons including these effects allows us to obtain any physical property of the QGP.

In Fig. 11 we show, in the $T-\mu_{\mathrm{B}} / T$ plane and at the chemical equilibration time $t_{\mathrm{ch}}$ of light quarks and gluons, i.e. for $\gamma_{\mathrm{q}}=\gamma_{\mathrm{G}}=1, \gamma_{\mathrm{s}}=0.15$, the QGP trajectories at constant energy per baryon (solid curves), beginning with $E / B=2.6$ (right curve), rising in increments of $1 \mathrm{GeV}$ up to $E / B=10.6 \mathrm{GeV}$ (left curve). Along these lines the QGP pressure varies. Assuming that the stopping of energy and baryon number is practically the same $\eta_{\mathrm{E}} \simeq \eta_{\mathrm{B}}$ we have $E_{\mathrm{CM}}=E / B$. One can now find points of pressure balance implied by Eq. (3) for each CM energy, which are shown by the dashed lines in Fig. 11. The highest assumes $\eta \equiv \eta_{\mathrm{p}}=\eta_{\mathrm{E}}=\eta_{\mathrm{B}}=1$, the lowest is for $\eta=0$ (vacuum pressure only), in increments of 0.25 . The intersections of these lines correspond to the temperatures $T_{\mathrm{ch}}$ attained at the end of the nuclear collision and before the transverse expansion and most of strangeness production occur.

\section{Temperature evolution in the collision}

We now consider in more detail the different stages of the nuclear collision. The particles observed in the collision are emanating at some low, freeze-out temperature $T_{\mathrm{f}}$. Even though the spectra of high $m_{\perp}$ strange and non-strange-particles are showing the same slope [7, 8], it cannot be assumed that they have frozen out at the same time, and hence it is convenient to introduce the strange-particle freeze-out temperature $T_{\mathrm{s}}$. This can be the case, since $T_{\mathrm{f}} \leq T_{\mathrm{s}}$ could be indeed much smaller than the temperature $T_{0}$ visible in the transverse particle spectra: the collective flow velocity $v_{\mathrm{f}}, v_{\mathrm{s}}$ superposes a Doppler blue shift with:

$$
T_{\mathrm{f}}=\sqrt{\frac{1-v_{\mathrm{f}}}{1+v_{\mathrm{f}}}} T_{0}, \quad T_{\mathrm{s}}=\sqrt{\frac{1-v_{\mathrm{s}}}{1+v_{\mathrm{s}}}} T_{0} .
$$

One finds $T_{0} \simeq 232 \mathrm{MeV}$ for both strange and non-strange-particles at high $m_{\perp}$, where other effects that distort the spectra (e.g. resonance disintegration) become negligible. This observed temperature $T_{0}$ is representative of the conditions of the fireball prior to its expansion after the initial compression, but after strangeness flavour was produced. 
We have already introduced two other temperatures: $T_{\mathrm{ch}}$ is the temperature reached in the system after the chemical equilibration of light quarks and gluons, but prior to the significant saturation of the strangeness phase-space, the production of strangeness being slower than of light quarks. The temperature $T_{\text {th }}$ is the temperature at which we can hypothesize that thermal motion is established already, while the valence quarks, gluons and quark-antiquark pairs produced are still far from chemical equilibrium. We thus have the following temperature hierarchy. Here the time increases from left to right while temperature decreases:

$$
T_{\text {th }}>T_{\text {ch }}>T_{0} \geq T_{\mathrm{s}} \geq T_{\mathrm{f}}
$$

The first inequality is related to cooling during the approach to chemical equilibrium (number equilibrium) of $q, \bar{q}, G$, the second one is due to the cooling while strangeness is produced and reaches its chemical equilibrium, and the last two arise from transverse expansion in which thermal energy is transferred to the collective flow.

We are thus equipped with a hierarchy of times and temperatures and a fully constrained time evolution scenario - for a given set of three chemical equilibration parameters $\gamma_{\mathrm{G}}, \gamma_{\mathrm{q}}, \gamma_{\mathrm{s}}$ there are in principle two unknown statistical parameters, $T$ and $\lambda_{q}$, which are determined after the equations of state and with two additional constraints: in our case, either given $E / B$ and total pressure $P=P_{\text {dyn }}+P_{\text {vac }}$, or fixed $E / B$ and $\lambda_{\mathrm{q}}$. We have already given the resulting values of $T_{\mathrm{ch}}$ in Fig. 1. In Fig. 2 2 we show the effect of cooling due to the formation of strange-quark pairs. We show the quantity $\Delta T=T_{\mathrm{ch}}-T_{0}$ as a function of $E / B$, solid line is for full stopping, the dashed one for $\eta_{i}=0.5$. These results were obtained by computing at a given $E / B$ and the suitably associated fixed $\lambda_{\mathrm{q}}$, as implied by the results of Fig. 1, and increasing the value of $\gamma_{\mathrm{s}}$ from 0.15 to 1 . Similarly, we can compute from the values $T_{\mathrm{ch}}$ the rise to $T_{\text {th }}$ by exploring the effect of reducing $\gamma_{\mathrm{G}}, \gamma_{\mathrm{q}}$ at constant pressure, but since the value of $T_{\text {th }}$ results form a chosen initial set of values for the pre-equilibrium occupancy factors, we will not discuss in detail the resulting first thermal temperature, except to say that it is up to $150 \mathrm{MeV}$ greater than $T_{\mathrm{ch}}$.

This hierarchy of thermal conditions in the fireball can be seen for the cases of particular interest in table 1. In the first column we show the assumed values of the chemical occupancies corresponding to the different stages. In the second, we list the properties we shall present explicitly (when possible these are given per baryon, with $\rho / \rho_{0}$ being the baryon density in units of the equilibrium nuclear density). In $3 \mathrm{rd}, 4$ th and 5 th columns we give our results for the three cases of specific interest: first for the $\mathrm{Si} \rightarrow \mathrm{Au}$ and $\mathrm{Au} \rightarrow \mathrm{Au}$ collisions at $E_{\mathrm{CM}}=2.6 \mathrm{GeV}, \eta_{i}=1$ (corresponding to the condition at BNL-AGS with 11-15 GeV A projectiles), then for the case of $\mathrm{S} \rightarrow \mathrm{W} / \mathrm{Pb}$ at $E_{\mathrm{CM}}=8.8 \mathrm{GeV}, \eta_{i}=0.5$ (corresponding to $\mathrm{S}$ projectile penetrating a tube of matter in a central impact on a heavy target at $200 \mathrm{GeV} \mathrm{A}$ ) and finally $\mathrm{Pb} \rightarrow \mathrm{Pb}$ collisions at $E_{\mathrm{CM}}=8.6 \mathrm{GeV}, \eta_{i}=1$ (corresponding to $\mathrm{Pb}$ projectiles at $157 \mathrm{GeV}$ A colliding at small impact parameter with $\mathrm{Pb}$ target).

In the first, top part of table 1, we present the most hypothetical results, as it corresponds to the earliest 'th' period, just when we can apply statistical methods and speak of temperature. Thus the value of the temperature $T_{\text {th }}$ shown there is a direct consequence of the number of gluons and light antiquarks assumed here to be present (note that we always make sure that for each stopped baryon there are three quarks per baryon in the fireball). Next, in the second part of the table comes the epoch, beginning with the temperature $T_{\mathrm{ch}}$, 
Table 1: Conditions in different collision systems at different evolution stages.

\begin{tabular}{|c|c|c|c|c|}
\hline \multirow{3}{*}{$\begin{array}{c}\text { Phase- } \\
\text { space } \\
\text { occupancy }\end{array}$} & \multirow{3}{*}{$\begin{array}{c}<s-\bar{s}>=0 \\
\lambda_{\mathrm{s}} \equiv 1\end{array}$} & \multicolumn{3}{|c|}{$E / B[\mathrm{GeV}]$} \\
\hline & & 2.6 & 8.8 & 8.6 \\
\hline & & $\begin{array}{c}\eta=1 \\
\mathrm{Au}-\mathrm{Au}\end{array}$ & $\begin{array}{c}\eta=0.5 \\
\mathrm{~S}-\mathrm{Pb}\end{array}$ & $\begin{array}{c}\eta=1 \\
\mathrm{~Pb}-\mathrm{Pb}\end{array}$ \\
\hline \multirow{4}{*}{$\gamma_{\mathrm{q}}=0.2$} & $T_{\text {th }}[\mathrm{GeV}]$ & 0.260 & 0.410 & 0.471 \\
\hline & $\lambda_{\mathrm{q}}$ & 9.95 & 1.78 & 2.00 \\
\hline & $n_{\mathrm{G}} / B$ & 0.20 & 1.55 & 1.25 \\
\hline & $n_{\mathrm{q}} / B$ & 3.00 & 5.12 & 3.77 \\
\hline \multirow[t]{3}{*}{$\gamma_{\mathrm{G}}=0.2$} & $n_{\overline{\mathrm{q}}} / B$ & 0.00 & 2.12 & 0.77 \\
\hline & $n_{\overline{\mathrm{s}}} / B$ & 0.02 & 0.16 & 0.13 \\
\hline & $P_{\mathrm{th}}\left[\mathrm{GeV} / \mathrm{fm}^{3}\right]$ & 0.46 & 0.79 & 1.46 \\
\hline \multirow{2}{*}{$\gamma_{\mathrm{s}}=0.03$} & $\rho / \rho_{0}$ & 3.34 & 1.70 & 3.18 \\
\hline & $S / B$ & 11.8 & 40.0 & 33.4 \\
\hline \multirow{4}{*}{$\gamma_{\mathrm{q}}=1$} & $T_{\mathrm{ch}}[\mathrm{GeV}]$ & 0.212 & 0.280 & 0.324 \\
\hline & $\lambda_{\mathrm{q}}$ & 4.16 & 1.49 & 1.61 \\
\hline & $n_{\mathrm{G}} / B$ & 0.56 & 2.50 & 2.08 \\
\hline & $n_{\mathrm{q}} / B$ & 3.11 & 5.16 & 4.62 \\
\hline \multirow[t]{3}{*}{$\gamma_{\mathrm{g}}=1$} & $n_{\overline{\mathrm{q}}} / B$ & 0.11 & 2.16 & 1.62 \\
\hline & $n_{\overline{\mathrm{s}}} / B$ & 0.05 & 0.25 & 0.21 \\
\hline & $P_{\mathrm{ch}}\left[\mathrm{GeV} / \mathrm{fm}^{3}\right]$ & 0.46 & 0.79 & 1.46 \\
\hline \multirow[t]{2}{*}{$\gamma_{\mathrm{s}}=0.15$} & $\rho / \rho_{0}$ & 3.35 & 1.80 & 3.19 \\
\hline & $S / B$ & 12.3 & 41.8 & 34.9 \\
\hline & & $\gamma_{\mathrm{s}}=1$ & $\gamma_{\mathrm{s}}=0.8$ & $\gamma_{\mathrm{s}}=1$ \\
\hline \multirow{3}{*}{$\gamma_{q}=1$} & $T_{0}[\mathrm{GeV}]$ & 0.190 & 0.233 & 0.270 \\
\hline & $\lambda_{\mathrm{q}}$ & 4.16 & 1.49 & 1.61 \\
\hline & $n_{\mathrm{G}} / B$ & 0.56 & 2.50 & 2.09 \\
\hline \multirow[t]{2}{*}{$\gamma_{\mathrm{G}}=1$} & $n_{\mathrm{q}} / B$ & 3.11 & 5.12 & 4.60 \\
\hline & $n_{\bar{q}} / B$ & 0.11 & 2.12 & 1.60 \\
\hline \multirow{2}{*}{$\begin{array}{c}\gamma_{\mathrm{s}}=0.8 \\
\text { or }\end{array}$} & $n_{\overline{\mathrm{s}}} / B$ & 0.28 & 1.27 & 1.07 \\
\hline & $P_{0}\left[\mathrm{GeV} / \mathrm{fm}^{3}\right]$ & 0.33 & 0.47 & 0.84 \\
\hline \multirow[t]{2}{*}{$\gamma_{\mathrm{s}}=1$} & $\rho / \rho_{0}$ & 2.41 & 1.05 & 1.81 \\
\hline & $S / B$ & 14.1 & 49.5 & 41.7 \\
\hline
\end{tabular}

when the fireball reaches chemical equilibrium for light quarks and gluons, the penetration of nuclei ends, and the expansion and equilibration of strangeness abundance commences. In the last, bottom part, we consider the properties after strangeness has been produced, for two slightly different values of $\gamma_{\mathrm{s}}$. As suggested by the $\mathrm{S} \rightarrow \mathrm{W}$ results [3] , we allow a a slight deviation from the equilibrium in this case. We recall that in the transverse spectra the temperature $T_{0}$ is visible at high $m_{\perp}$ and we see that the statistical properties determined 
here are in remarkable agreement with the values extracted from experiments: $T_{0}=232 \pm 5$ $\mathrm{MeV}, \lambda_{\mathrm{q}}=1.48 \pm 0.05$ [3].

\section{Some experimental consequences}

When looking at the $\mathrm{Au}-\mathrm{Au}$ predictions at the lowest energy considered here (assuming QGP formation), the first and most striking result is that, at some early stage (first part of table 1), there are practically only valence quarks present practically no noticeable pair or glue production has taken place. Even when we consider the chemically equilibrated system at later time, we see that the glue abundance is just equal to the number of $s$ and $\bar{s}$ quarks. This relatively small number of gluons may be inhibiting the production of strangeness, and thus the assumed strangeness chemical equilibrium, even if QGP were formed in these collisions, is probably over-optimistic. Because of the relatively large expected value of $\lambda_{\mathrm{q}}$, a simple test for QGP in this system is the measurement of the ratios of particles such as $\bar{\Lambda} / \bar{p} \simeq \overline{\Xi^{-}} / \bar{\Lambda} \propto \gamma_{\mathrm{s}} \lambda_{\mathrm{q}}$, which should thus considerably exceed expectations [5]. Also note that our earlier analysis of the $\mathrm{Si}-\mathrm{Au}$ results 5 suggested that, should QGP be formed in the $14 \mathrm{GeV}$ A interactions, its disintegration would require a considerable re-equilibration in the final state, since $\lambda_{\mathrm{s}} \simeq 1.7$ was found in the final state, and not $\lambda_{\mathrm{s}} \simeq 1$ as expected in rapid QGP dissociation. The necessary presence of the intermediate re-equilibration era complicates the arguments for, or against, possible formation of QGP in these collisions. We further note that the production of exotic strange matter containing an unusual strangeness fraction is difficult, and indeed unlikely, since $s / q \simeq 0.09$. We also note that even though this ratio rises to 0.25 at the higher energies, there is so much more entropy produced there that it is hard to see how the system could cool down without dissociating by evaporating the excess entropy. Our results thus do not encourage searches for strange matter exotica in relativistic heavy-ion collisions.

We next consider, in table 1 , the subtle differences between the $\mathrm{S} \rightarrow \mathrm{Pb}$ and $\mathrm{Pb} \rightarrow \mathrm{Pb}$ collisions. We predict a rise in temperature and $\lambda_{\mathrm{q}}$ - the observable temperature rises from $T_{0}=233 \mathrm{MeV}$ to $T_{0}=270 \mathrm{MeV}$, which should indeed be easily visible in the high $m_{\perp}$-spectra. Because of the considerable increase in baryon density (from $1.8 \rho_{0}$ to $3.2 \rho_{0}$ ) we see a noticeable drop in specific entropy. This is intuitively correct, since the energy per baryon is similar; the greater energy content per final-state particle at higher temperature thus entails a smaller number of particles per baryon, and hence a smaller entropy content per baryon. We also note that even the slight rise in $\lambda_{\mathrm{q}}$ has a considerable impact on the ratio $\bar{\Lambda} / \Lambda$, which is reduced by the factor $(1.49 / 1.61)^{4}=0.73$ due primarily to a greater number of $\Lambda$, given the greater baryon density achieved. The specific yield of strange-particles is also expected to drop, but it remains relatively high as the number of $\bar{s}$ is greater than the number of $\bar{u}$ or $\bar{d}$ (note that $\bar{q}=\bar{u}+\bar{d}$, and naturally $\bar{s}=s$ ). Thus the ratios of particles such as $\overline{\Xi^{-}} / \bar{\Lambda}$, which are considered as a test for the QGP phase [19, [13], will increase by $35-50 \%$ as we move to the $\mathrm{Pb} \rightarrow \mathrm{Pb}$ system from $\mathrm{S} \rightarrow \mathrm{Pb}$. Interestingly, this ratio will modestly increase while collision energy decreases, as long as the QGP phase is formed, due to an increase in the value of $\lambda_{\mathrm{q}}$, which we predict. A sudden decrease should follow, when $E_{\mathrm{CM}}$ drops below the threshold for the formation of QGP.

We now comment on the consequences of the relatively high values of $T_{\text {ch }}=280 \mathrm{MeV}$ 
$(\mathrm{S} \rightarrow \mathrm{Pb}$ case $)$ and $T_{\mathrm{ch}}=324 \mathrm{MeV}(\mathrm{Pb} \rightarrow \mathrm{Pb}$ case $)$ and even greater pre-chemical-equilibrium values reported in table 1 . A calculation of the dileptons yields in similar conditions was already performed [20], and we have interpolated these results to the value of temperature applicable for an $\mathrm{S} \rightarrow \mathrm{Pb}$ collision. In Fig. 3 we show (solid line) as a function of the dimuon invariant mass the sum of the thermal QGP dimuons (short-dashed contribution), the hadron contribution (long-dashed component) and the Drell-Yan (with $K=2$ ) together with renormalized $J / \psi$ contributions (dotted line, chosen to fit the $J / \psi$ peak). The relative yield of the QGP radiance, in the particularly interesting region between 1.4 and $2.6 \mathrm{GeV}$ of the dimuon invariant mass, arises from these normalizations and is hence not arbitrary. This result is in agreement with the experimental ones results presented recently [21] and shown here as open squares. While the agreement in slope between the 1.4 and $2.6 \mathrm{GeV}$ dimuon invariant mass is due to the magnitude of the source temperature, the agreement in normalization must be seen as being probably a happy coincidence: the strength of the source is roughly proportional to the product of the abundance of quarks and antiquarks, which is dominated by the valence quarks, see table 1. However, the theoretical dilepton work 20] did not allow for any stopping of baryon number.

The rise in temperature, seen as we move from the $\mathrm{S} \rightarrow \mathrm{Pb}$ to the $\mathrm{Pb} \rightarrow \mathrm{Pb}$ system, suggests a greater visibility of photons and dileptons in the heavier system. However, the situation is more complex: the rate of signal to noise of photons and dileptons is proportional to $n_{\mathrm{q}} n_{\overline{\mathrm{q}}} / S$, which drops by $20 \%$ as we move from $\mathrm{S} \rightarrow \mathrm{W} / \mathrm{Pb}$ to $\mathrm{Pb} \rightarrow \mathrm{Pb}$ collisions. Thus while the invariant-mass region of current interest could appear flatter due to higher temperature, the relative strength of the signal to hadronic background rate will be a bit less. More vexing is that this very interesting signal is much less specific and sensitive to the source conditions - we hope to return in the near future to a more detailed study of the dilepton and photon spectra using the evolution, as determined here, of the QGP phase.

\section{Final remarks}

Another important result to observe in table 1 is that the entropy content, which determines the final-particle multiplicity [2, 3], evolves very little and is to all purposes already present at the initial stage, when quarks and gluons are still far from abundance equilibrium [17]. Practically all the rise we present is due to the formation of the strange flavour against the background of the thermalized and expanding quark-gluon fireball. We have an enormous amount of strangeness, as the tables show, and hence the fact that $20 \%$ of entropy is due to strangeness production is not surprising. In total for the $\mathrm{S} \rightarrow \mathrm{Pb}$ and $\mathrm{Pb} \rightarrow \mathrm{Pb}$, there are $n / B=0.25 S / B$ particles per baryon, once chemical equilibrium is reached, in agreement with the 'rule' that in a relativistic non-degenerate system on average, each particle carries $\simeq 4$ units of entropy. The $\mathrm{Au} \rightarrow \mathrm{Au}$ system is essentially a degenerate quark matter and one finds therefore less entropy, since only quarks at the Fermi surface contribute. The entropy per particle drops, and hence the coefficient given above is greater $(0.25 \rightarrow 0.32)$.

Our present results reconfirm the key message regarding entropy [17], which is that it is produced during the thermalization phase, and not during the approach to chemical equilibrium when most particles are produced. Thus the final hadronic multiplicity is determined in the initial instants of the collision, and is not determined by model calculations, which 
assume initial condition.

As the system evolves towards the final freeze-out conditions, its entropy content remains in essence unchanged, as we have so far discovered no entropy-generating mechanisms acting at this very last stage of the evolution. Thus, irrespective of the values of freeze-out temperatures $T_{\mathrm{s}}$ and $T_{\mathrm{f}}$ at which the strange and, respectively, non-strange particles decouple, the specific entropy content of the hadronic system is expected to remain the same. Interpreting particle abundances in terms of chemical potentials and temperature of a source, and assuming an equation of state, the entropy content can be calculated. For the $\mathrm{S} \rightarrow \mathrm{W}$ collisions at $200 \mathrm{GeV}$ A we found earlier [2, 3] that for the QGP equations of state we should expect 50 units of entropy per baryon in the final state, as we now also find in an ab initio calculation, as shown in table 1. If the (multi-)strange (anti-)baryons [7, 11], which served us in the determination of the properties of the fireball, were to originate from a confined hadronic state, with properties described by a mixture of hadronic resonances (Hagedorn gas), we find an entropy content that is nearly half as large as that of a QGP source. At a late time in collisions all hadrons have materialized. Unless some new physics is introduced that generates entropy in the evolution of the hadronic matter, in particular after strange-particles were produced from such a hadron gas, the situation is that the expected final particle multiplicity must be very different for the two evolution scenarios, i.e. HG or QGP. Studying the hadronic multiplicity, we have determined that it implies an entropy-rich source [2, 3].

In the present work we have shown that this entropy-rich state is governed by the equations of state of the perturbative QGP. In particular we found (see table 1) in an ab initio calculation that when (nearly) full chemical equilibrium is reached, the apparent source temperature and baryo-chemical conditions are as deduced from the analysis of the strangeparticle data for $200 \mathrm{GeV}$ A collisions. We have also demonstrated that the observed excess of dileptons in the invariant mass range $1.4-2.6 \mathrm{GeV}$ is consistently described by our QGP fireball model. We have explored the properties of a QGP fireball formed possibly in the $\mathrm{Au} \rightarrow \mathrm{Au}$ collisions at $11 \mathrm{GeV} \mathrm{A}$ and we have also made detailed predictions about the conditions expected in the forthcoming $\mathrm{Pb} \rightarrow \mathrm{Pb}$ collisions at $200 \mathrm{GeV} \mathrm{A}$.

\section{Acknowledgement}

J. R. acknowledges partial support from the US DOE grant DE-FG02-92ER40733.

A. T. wishes to thank Siki Ibn Mouloud for his poetic considerations on Strangeness. 


\section{References}

[1] J. Letessier, J. Rafelski and A. Tounsi, Phys. Lett. B323 (1994) 393.

[2] J. Letessier, A. Tounsi, U. Heinz, J. Sollfrank and J. Rafelski, Phys. Rev. Lett. 70 (1993) 3530 .

J. Rafelski, J. Letessier and A. Tounsi, in Proceedings of the XXVI International Conference on High Energy Physics, Dallas, Texas, 1992, AIP Conference Proceedings No 272, 983, J.R. Sanford, Editor.

[3] J. Letessier, A. Tounsi, U. Heinz, J. Sollfrank and J. Rafelski, Strangeness Conservation in hot nuclear fireballs, Paris preprint LPTHE/92-27R, submitted to Phys. Rev. D.

U. Heinz, Nucl. Phys. A566 (1994) 205c.

[4] J. Letessier, J. Rafelski and A. Tounsi, Phys. Lett. B321 (1994) 394.

[5] J. Letessier, J. Rafelski and A. Tounsi, Strangeness and particle freeze-out in nuclear collisions at 14.6 GeV A, Paris preprint LPTHE/94-15, Phys. Lett. B, in press.

[6] E. Schnedermann, J. Sollfrank and U. Heinz, Fireball Spectra, in: Particle Production in Highly Excited Matter, H. H. Gutbrod and J. Rafelski, eds., NATO Physics series Vol. B 303, Plenum Press, New York, 1993, 175.

[7] D. Evans et al. (WA85 collaboration), Nucl. Phys. A566 (1994) 225c;

S. Abatzis et al. (WA85 collaboration), Phys. Lett. B259 (1991) 508;

S. Abatzis et al. (WA85 collaboration), Phys. Lett. B270 (1991) 123;

[8] R. Santo et al. (WA80 collaboration), Nucl. Phys. A566 (1994) 61c.

[9] J. Rafelski, Phys. Lett. B262 (1991) 333;

J. Rafelski, Nucl. Phys. A544 (1992) 279c, and references therein.

[10] J. Sollfrank, M. Gaździcki, U. Heinz and J. Rafelski, Regensburg preprint TPR-93-14, Z. Physik $\mathbf{C}$, in press.

[11] S. Abatzis et al. (WA85 collaboration), Phys. Lett. B316 (1993) 615.

[12] J. Rafelski and M. Danos, Strangeness flow differences in nuclear collisions at 15 and $200 \mathrm{GeV}$ A, Phys. Rev. C, in press.

[13] J. Rafelski, On the trail of QGP: Strange Antibaryons in Nuclear Collisions, in: Particle Production in Highly Excited Matter, H. H. Gutbrod and J. Rafelski, eds, NATO Physics series Vol. B 303, Plenum Press, New York, 1993, 529, and references there in.

[14] G.W. London, Result from the CERN Pilot Study of Ultra-Relativistic Nucleus-Nucleus Interaction, in: proceedings of the VIII International Conference on Physics in Collision, Capri, 1988, P. Strolin, ed., Editions Frontières, Paris, 1989, 397.

I. Otterlund, Physics of Relativistic Nuclear Collisions, in: Particle Production in Highly 
Excited Matter, H. H. Gutbrod and J. Rafelski, eds, NATO Physics series Vol. B 303, Plenum Press, New York, 1993, 57.

S.P. Sorensen et al. Nuclear Stopping Power, in: Proc. XXI Int. Symposium on Multiparticle Dynamics 1991, World Scientific, Singapore, 1992.

[15] K. Geiger and B. Müller, Nuc. Phys. B369 (1992) 600;

K. Geiger, Phys. Rev. D46 (1992) 4965, 4986.

[16] L. Xiong and E. Shuryak, Phys. Rev. C49 (1994) 2203.

[17] J. Letessier, J. Rafelski and A. Tounsi, Gluon production, cooling and entropy in nuclear collisions, Phys. Rev. C, in press.

[18] S.R. de Groot, W.A. van Leeuwen, Ch.G. van Weert, Relativistic Kinetic Theory, North Holland, Amsterdam, 1980.

[19] J. Rafelski, Phys. Rep. 88 (1982) 331.

[20] J. Letessier and A. Tounsi, Phys. Rev. D40 (1989) 2914;

M. Kataja, J. Letessier, P.V. Ruuskanen and A. Tounsi, Z. Physik C55 (1992) 153.

[21] M.A. Mazzani (Helios3 collaboration), Nucl. Phys. A566 (1994) 95c.

\section{FIGURE CAPTIONS}

Figure 1: The $T-\mu_{\mathrm{B}} / T$ plane. solid lines: trajectories at constant energy per baryon in QGP, with $E / B=2.6$ up to $10.6 \mathrm{GeV}$ (rising in intervals of $1 \mathrm{GeV}$ from right to left); dashed lines: lines of constant pressure $\mathrm{P}$ in QGP with the value of $\mathrm{P}$ determined by Eq. (3) for any given $E / B$, with $\eta_{\mathrm{p}}=0$ up to 1 (rising in intervals of 0.25 with $\eta$ ).

Figure 2: $\quad \Delta T=T_{\mathrm{ch}}-T_{0}$ as function of $E / B$, obtained at fixed $\lambda_{\mathrm{q}}$ with $\gamma_{\mathrm{s}}$ changing from 0.15 to 1 . Solid line $\eta_{\mathrm{p}}=1$, dashed line $\eta_{\mathrm{p}}=0.5$.

Figure 3: Spectrum of dimuons as a function of the dimuon invariant mass (arbitrary normalization), after Ref. [20]. The solid line is the sum of the thermal QGP dimuons (shortdashed contribution), the hadron contribution (long-dashed component) and the Drell-Yan (with $K=2$ ) together with normalized $J / \psi$ contributions (dotted line, chosen to fit the $J / \psi$ peak). Experimental results (open squares) are read from Fig. 5, in [21]. 


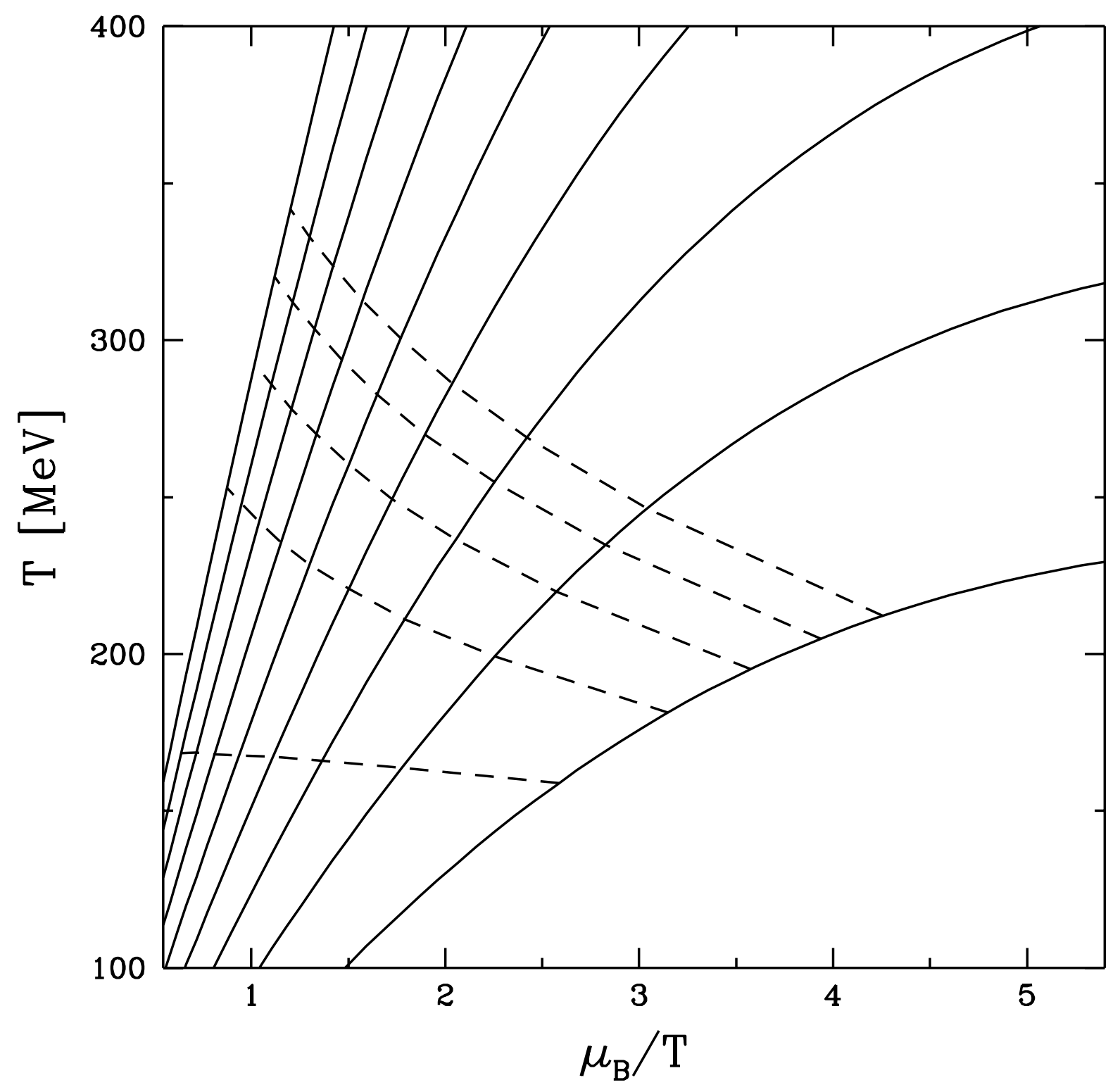

FIGURE 1 


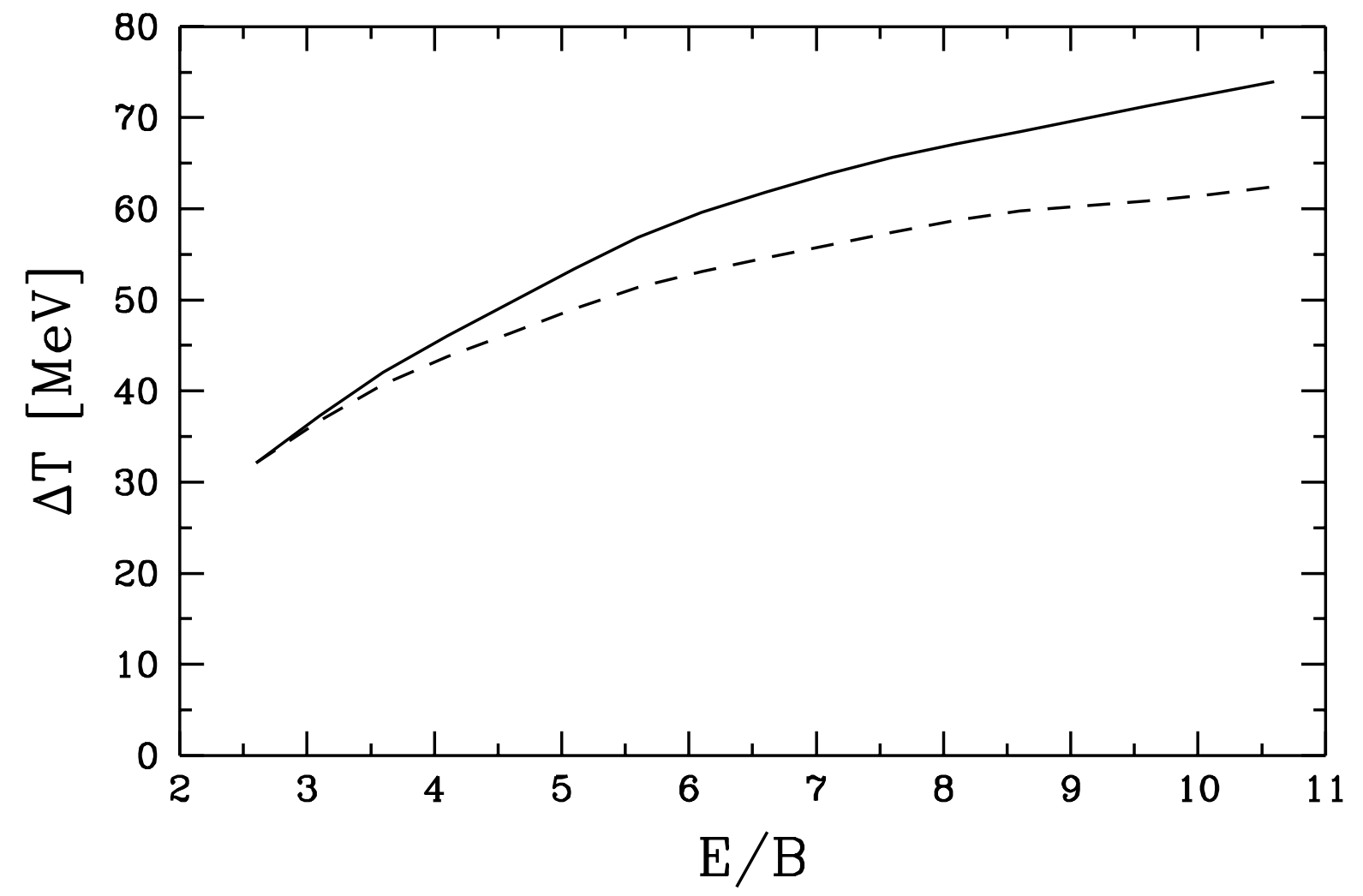

FIGURE 2 


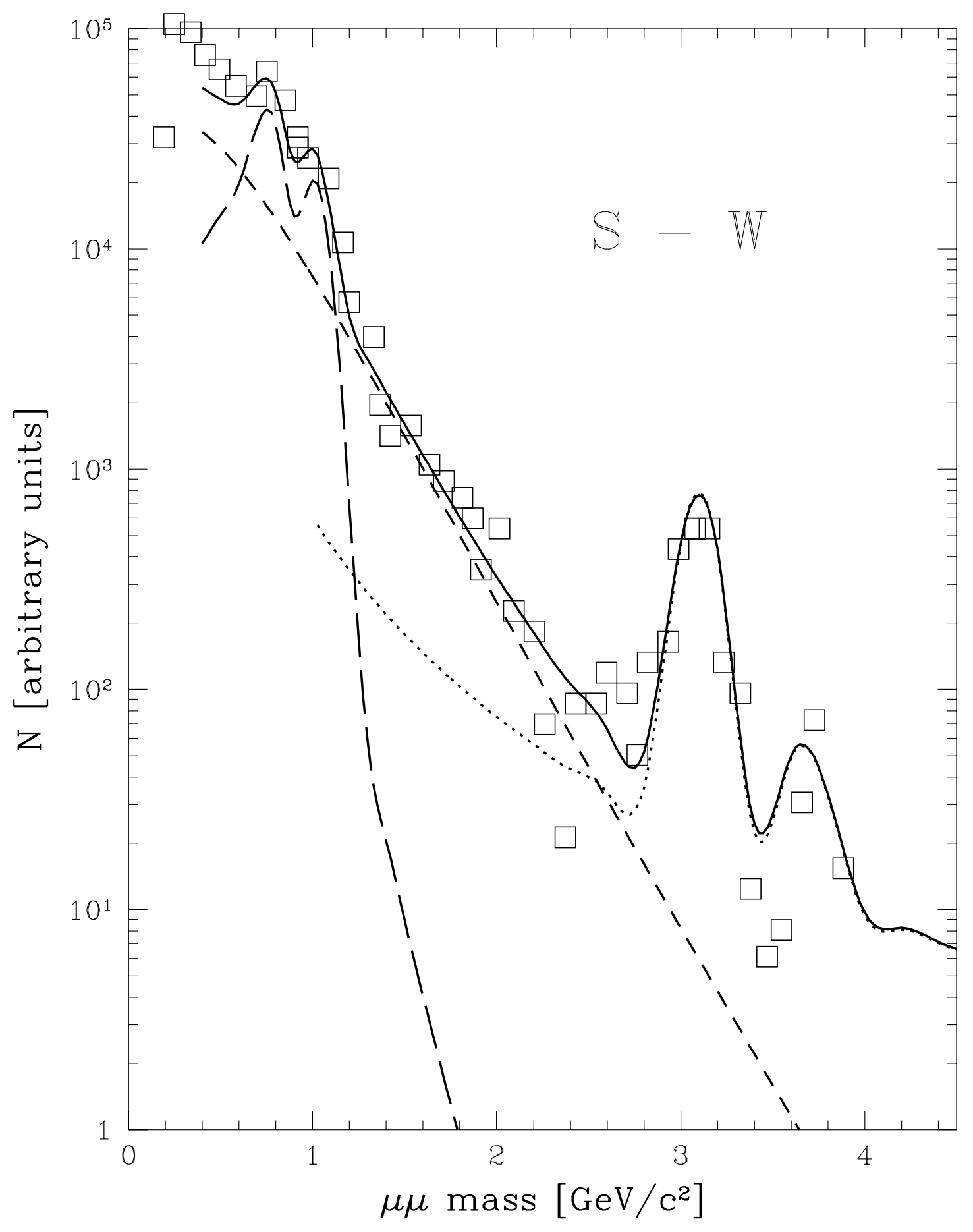

FIGURE 3 